\title{
REGULARITY OF THE DISTANCE FUNCTION TO SMOOTH HYPERSURFACES IN SOME TWO-STEP CARNOT GROUPS
}

\author{
Nicola Arcozzi*, Fausto Ferrari ${ }^{\dagger}$ and Francescopaolo Montefalcone ${ }^{\ddagger}$
}

\author{
Università degli Studi di Bologna, Dipartimento di Matematica \\ Piazza di P.ta S.Donato, 5, 40126 Bologna, Italy; nicola.arcozzi@unibo.it \\ Università degli Studi di Bologna, Dipartimento di Matematica \\ Piazza di P.ta S.Donato, 5, 40126 Bologna, Italy; fausto.ferrari@unibo.it \\ Università di Padova, Dipartimento di Matematica Pura e Applicata \\ Via Trieste, 63, 35121 Padova, Italy; montefal@math.unipd.it
}

\begin{abstract}
We study geometric properties of the Carnot-Carathéodory signed distance $\delta^{s}$ to a smooth hypersurface $S$ in some 2-step Carnot groups. In particular, a sub-Riemannian version of Gauss' Lemma is proved.
\end{abstract}

\section{Introduction and statement of the main results}

In this paper we study some fine properties of the Carnot-Carathéodory, signed, distance function to a hypersurface in some 2-step Carnot groups and their relation to the hypersurface's geometry. Given a 2-step Carnot group G, endowed with a fixed sub-Riemannian structure, we denote by $d$ the Carnot-Carathéodory distance in $\mathbf{G}$. If $S$ is a fixed hypersurface in $\mathbf{G}$, we denote by $\delta$ the function "distance from $S^{\prime \prime}$, that is

$$
\delta(p):=\inf \{d(p, q): q \in S\} \quad \forall p \in \mathbf{G} .
$$

We always tacitly assume that $S=\partial \Omega$ is the boundary of a connected, open subset of $\mathbf{G}$, such that $\partial \Omega=\partial(\mathbf{G} \backslash \Omega)$. We also consider the signed distance

$$
\delta^{s}(p):= \begin{cases}\delta(p) & \text { if } p \notin \Omega, \\ -\delta(p) & \text { if } p \in \Omega .\end{cases}
$$

In Section 2.1 we will recall basic notions on 2-step Carnot groups. However, in order to state our main results, we anticipate here some definitions.

For brevity, we call special all the 2-step Carnot groups G having the following smoothness property:

https://doi.org/10.5186/aasfm.2017.4222

2010 Mathematics Subject Classification: Primary 49Q15, 46E35, 22E60.

Key words: Carnot groups, sub-Riemannian geometry, CC-metrics, distance from hypersurfaces, metric normal, normal geodesics.

*Partially supported by University of Bologna, Italy, funds for selected research topics and by GNAMPA of INdAM, Italy.

${ }^{\dagger}$ Partially supported by GALA project Geometric Analysis in Lie groups and Applications, by the European Commission within the 6th Framework Programme, by PRIN project(MiUR) Metodi di viscosità, geometrici e di controllo per modelli diffusivi nonlineari, by the ERC starting grant project EPSILON (Elliptic Pdes and Symmetry of Interfaces and Layers for Odd Nonlinearities) 277749 and by University of Bologna, Italy.

${ }^{\ddagger}$ Partially supported by University of Bologna, Italy, funds for selected research topics, by GNAMPA of INdAM, by CIRM, Fondazione Bruno Kessler, and by the Fondazione CaRiPaRo Project "Nonlinear Partial Differential Equations: models, analysis, and control-theoretic problems". 
( $\star$ Let $d(\cdot, 0): \mathbf{G} \longrightarrow[0,+\infty[$ be the distance function to $0 \in \mathbf{G}$ and let $\gamma:[0, r[$ $\rightarrow \mathbf{G}$ be a length-minimizing geodesic connecting 0 with $p \in \gamma([0, r[)$. Then, $d(\cdot, 0)$ is of class $\mathbf{C}^{2}$ in a neighborhood of $\gamma(t)$ for any $t$ in $] 0, r[$.

Note that by the left translation invariance of the CC-distance, one may replace $0 \in \mathbf{G}$ by any other point $q \in \mathbf{G}$.

To the authors' knowledge, it is not known whether all 2-step Carnot groups have this property. We stress, however, that all 2-step Carnot groups that do not contain abnormal geodesics (see, for instance, $[2,13,21]$ ) have to satisfy hypothesis ( $\star$ ). More precisely, it follows from Theorem 10.15 in [2] (see also Proposition 10.11 and the results in Chapter 7.2) that if $\gamma:[0, r[\rightarrow \mathbf{G}$ is a length-minimizing geodesic connecting 0 with $p$, then the (squared) CC-distance function $d(\cdot, 0)$ to 0 is of class $\mathbf{C}^{\infty}$ in (a neighborhood of) $\gamma(t)$ for any $t$ in $] 0, r$. Moreover, it is known that abnormal geodesics do not exist at all for a subclass of 2-step Carnot groups called Métivier groups (hence, in all $\mathrm{H}$-type groups: in particular, this holds true in Heisenberg groups $\mathbf{H}^{n}$ ); see, for instance, [23]. We refer the reader to [1, 2, 15], for an exhaustive introduction and some recent results on this problem.

Let $S_{0}:=S \backslash C_{S}$ be the non-characteristic set of the $\mathbf{C}^{2}$ hypersurface $S$. Recall that a point $p \in S$ is a characteristic point if the tangent space to $S$ at $p$ contains the horizontal space at $p$. The set $C_{S}$ denotes the set of all characteristic points of $S$. We also need to introduce the following technical property concerning some sets $U \subset \mathbf{G}$ with respect to the hypersurface $S$. We say that the set $U \subset \mathbf{G}$ satisfies the unique nearest point property with respect to the hypersurface $S$ if for each $p$ in $U$ exactly one point $q$ in $S$ exists such that $d(p, q)=\delta(p)$.

Our main theorem is a regularity result for $\delta$.

Theorem 1.1. Let $\mathbf{G}$ be a special 2-step Carnot group. Let $S=\partial \Omega \subset \mathbf{G}$ be a hypersurface of class $\mathbf{C}^{k}$, with $k \geq 2$. Then, there exists an open subset $U$ of $\mathbf{G}$ having the unique nearest point property, with respect to the $C C$-distance $\delta$ to $S$, such that $U \cap S=S_{0}$, where $S_{0}$ denotes the non-characteristic set of $S$. In addition, the signed distance function $\delta^{s}$ is of class $\mathbf{C}^{k}$ on $U$.

In the special case of $\mathbf{H}^{1}$, Theorem 1.1 was proved in a slightly stronger form by the first two authors in [3] and [4]. The main tools in proving Theorem 1.1 are a sub-Riemannian version of the classical Gauss' Lemma (see [7]) and a metric exponential map. In order to describe them, we have to be more precise about the sub-Riemannian structure we are working with. The group $\mathbf{G}$ is a 2-step Carnot group: a $n$-dimensional real Lie group, whose Lie algebra $\mathfrak{g}$ (that is, the space of all left invariant vector fields on $\mathbf{G}$ ) admits a stratification $\mathfrak{g}=H \oplus V$, where $V:=[H, H]$ and $[H, V]=0$. The layers $H$ and $V$ are called horizontal and vertical, respectively. For more details we refer to Section 2.1. Let $\langle\cdot, \cdot\rangle_{H}$ be a fixed positive definite quadratic form on $H$, and let $\left\{X_{1}, \ldots, X_{h}\right\}$ be an orthonormal basis for $H$. It is well known that there is a sub-Riemannian distance associated with $\langle\cdot, \cdot\rangle_{H}$, and that the distance between two points is realized by the length of a geodesic. These notions are further detailed in Sections 2.1 and 2.2 of this paper. Fix now a basis $\left\{X_{h+1}, \ldots, X_{n}\right\}$ of $V$ and consider the left-invariant Riemannian metric $\langle\cdot, \cdot\rangle=\langle\cdot, \cdot\rangle_{H}+\langle\cdot, \cdot\rangle_{V}$, which makes $\left\{X_{1}, \ldots, X_{n}\right\}$ into an orthonormal basis of $\mathfrak{g}$.

By means of the Riemannian metric $\langle\cdot, \cdot\rangle$, we can identify vectors and covectors, and we shall do so throughout the article, unless explicitly stated.

Let $\mathcal{P}_{H}$ and $\mathcal{P}_{V}$ denote the orthogonal projection operators onto $H$ and $V$, respectively. If $\nu$ denotes the Riemannian unit normal vector to $S$ pointing outside $\Omega$, 
then for every $x \in S_{0}:=\left\{y \in S:\left|\mathcal{P}_{H} \nu(y)\right| \neq 0\right\}$ we set

$$
\varpi(x):=\frac{\mathcal{P}_{V} \nu(x)}{\left|\mathcal{P}_{H} \nu(x)\right|} \quad \text { and } \quad \mathcal{N}(x):=\frac{\nu(x)}{\left|\mathcal{P}_{H} \nu(x)\right|}=\left(\nu_{H}(x), \varpi(x)\right) .
$$

When necessary, we shall add a superscript to indicate the dependence on a particular hypersurface $S$. In the case of the Heisenberg group $\mathbf{H}^{1}$, the quantity $\varpi$ was introduced in [3] and [4] in connection with the distance to a smooth surface. In general, this quantity plays a special role in the theory of hypersurfaces endowed with the $H$-perimeter measure; see [18].

For any $\left(x_{0}, v_{0}\right) \in T \mathbf{G}$, with $x_{0} \in \mathbf{G}, v_{0} \in T_{x_{0}} \mathbf{G}$, such that $v_{0}=v_{H}^{0}+v_{V}^{0},\left|v_{H}^{0}\right|=1$, there exists a unique unit speed normal geodesic $x:[0, r[\rightarrow \mathbf{G}$ (for some $r>0$ ) such that: (i) $x(0)=x_{0}$; (ii) $x^{\prime}(0)=v_{H}^{0}$; (iii) the vector of "vertical parameters" of $x(t)$ at $t=0$ is $v_{V}^{0}$. More precisely, normal geodesics can be seen as (projection onto $\mathbf{G}$ of the) solutions $(x(t), v(t)) \in T \mathbf{G}$ to the system of ODEs

$$
\left\{\begin{array}{l}
x^{\prime}=v_{H}, \\
v_{H}^{\prime}=-C_{H}\left(v_{V}\right) v_{H}, \\
v_{V}^{\prime}=0,
\end{array}\right.
$$

where $C_{H}\left(v_{V}\right): H \rightarrow H$ denotes a linear map which only depends on the structure constants of the Lie algebra of $\mathbf{G}$ and (linearly) on the "vertical vector" $v_{V}=v_{V}^{0}$. (This definition can be easily deduced from the standard Hamiltonian formulation, for which we refer the reader to $[2,13,21,25])$.

We stress that in 2-step Carnot groups all geodesics are actually normal (though not necessarily "strictly normal"); see [13]. We use the solutions to (1) to define a sub-Riemannian exponential map

$$
\exp _{\mathcal{S R}}\left(x_{0}, v_{0}\right)(t):=x(t)
$$

with $x(t)$ solution to $(1)$.

Let $\mathbf{S}\left(x_{0}, t\right):=\left\{x \in \mathbf{G}: d\left(x, x_{0}\right)=t\right\}$ be the CC-sphere of radius $t>0$. In general, it is not known if $\mathbf{S}\left(x_{0}, t\right)$ is smooth, even at points $x(t) \in \mathbf{S}\left(x_{0}, t\right)$ which are joined with $x_{0}$ by a unique length-minimizing geodesic. If the group $\mathbf{G}$ is special this fact holds true by the Implicit Function Theorem. Let $\operatorname{grad}_{H}$ and $\operatorname{grad}_{V}$ denote the orthogonal projections of the Riemannian gradient grad onto the subspaces $H$ and $V$ of the Lie algebra $\mathfrak{g}$. The following result is the key tool to prove Theorem 1.1.

Theorem 1.2. (Gauss' Lemma) Let $\mathbf{G}$ be a special 2-step Carnot group and let $\gamma:[0, r[\rightarrow \mathbf{G}(r>0)$ be any (unit speed) length-minimizing normal geodesic with initial conditions $x_{0} \in \mathbf{G}, v_{0} \in T_{x_{0}} \mathbf{G}$, such that $v_{0}=v_{H}^{0}+v_{V}^{0},\left|v_{H}^{0}\right|=1$. Let $d(x):=d\left(x_{0}, x\right)$ for any $x \in \mathbf{G}$. Then, the following holds:

(i) $\gamma^{\prime}(t)=\operatorname{grad}_{H} d(\gamma(t)) \quad \forall t \in(0, r) \quad$ "Horizontal Gauss' Lemma";

(ii) there exists a skew-symmetric $(h \times h)$-matrix $C_{H}\left(\varpi^{\mathbf{s}\left(x_{0}, t\right)}\right)$, which only depends on the structure constants of the Lie algebra and (linearly) on the vector $\varpi^{\mathrm{S}\left(x_{0}, t\right)}$, such that

$$
\left.\frac{d}{d t} \operatorname{grad} d(\gamma(t))=-C_{H}\left(\varpi^{\mathbf{s}\left(x_{0}, t\right)}(\gamma(t))\right) \operatorname{grad}_{H}(\gamma(t)) \quad \forall t \in\right] 0, r[
$$

"Vertical Gauss' Lemma". 
Here, the symbol $\varpi^{\mathbf{S}\left(x_{0}, t\right)}$ denotes the normalized vertical projection of the Riemannian unit normal to the CC-sphere $\mathbf{S}\left(x_{0}, t\right)$; see Definition 2.2. We also remark that, a posteriori, one has $\varpi^{\mathbf{S}\left(x_{0}, t\right)}(x)=\operatorname{grad}_{V} d\left(x_{0}, x\right)$ at any smooth point $x \in \mathbf{S}\left(x_{0}, t\right)$.

By the very definition of $\varpi^{S}$ and $\varpi^{\mathbf{S}\left(x_{0}, t\right)}$, if a CC-sphere $\mathbf{S}\left(x_{0}, t\right)$ touches a regular hypersurface $S$ at a non-characteristic point $x_{1}$, then we have that $\varpi^{\mathrm{s}\left(x_{0}, r\right)}\left(x_{1}\right)=$ $\pm \varpi^{S}\left(x_{1}\right)$, as the vector $\varpi^{S}$ only depends on the Riemannian unit normal vector, which is common to both hypersurfaces at the point $x_{1}$.

This simple observation is crucial. Indeed, together with Gauss' Lemma, it implies that the Riemannian normal to $S$ at a non-characteristic point $x_{0}$ determines, via calculation of $\varpi^{S}$, the (unique) length-minimizing normal geodesic $\gamma(t)$, which starts at $\gamma(0)=x_{0}$ and passes through $x_{1}$. On the other hand, if $x_{1} \in S$ is non characteristic, we prove that there exists a CC-sphere $\mathbf{S}(\gamma(t), t)$ that touches $S$ at $x_{1}$ (that is, $T_{x_{1}} S=T_{x_{1}} \mathbf{S}(\gamma(t), t)$ ). Observe also that $\varpi^{S}$ depends on the particular Riemannian extension $\langle\cdot, \cdot\rangle$ of the sub-Riemannian metric $\langle\cdot, \cdot\rangle_{H}$, but the geodesic $\gamma$, and its metric properties, do not.

Gauss' Lemma allows us to define the following metric exponential map

$$
\exp _{S}: S_{0} \times \mathbf{R} \rightarrow \mathbf{G}, \quad \exp _{S}(y, t):=\exp _{\mathcal{S R}}(y, \mathcal{N}(y))(t) .
$$

Let now $y_{0} \in S_{0}=S \backslash C_{S}$ be a non characteristic point and let us introduce a system of Riemannian normal coordinates $u \equiv\left(u_{1}, \ldots, u_{n-1}\right)$ around $y_{0}$; see Section 3.2. Thus, if $y(u) \equiv y\left(u_{1}, \ldots, u_{n-1}\right)$ describes a neighborhood $U_{0} \subset S_{0}$ of $y_{0}$, we henceforth set

$$
\Phi(u, t) \equiv \Phi\left(u_{1}, u_{2} \ldots, u_{n-1}, t\right):=\exp _{S}(y(u), t)
$$

where $\left.\Phi: \widetilde{U_{0}} \times\right]-\epsilon, \epsilon\left[\longrightarrow \mathbf{G}\right.$ and $\widetilde{U_{0}} \subset \mathbf{R}^{n-1}$ denotes an open neighborhood of $0_{\mathbf{R}^{n-1}}$. Note that $\Phi\left(0_{\mathbf{R}^{n-1}}, 0\right)=\exp _{S}\left(y_{0}, 0\right)=y_{0}$. The function $\Phi$ has an intrinsic meaning described in the following result, whose proof is given in Section 3.2.

Theorem 1.3. Let $\mathbf{G}$ be a 2-step Carnot group. Let $S \subset \mathbf{G}$ be a hypersurface of class $\mathbf{C}^{k}$, with $k \geq 2$, and let $y_{0} \in S_{0}$ and $\Phi$ be as above. Then

$$
\left|\operatorname{det}\left[\mathcal{J}_{\left(0_{\mathbf{R}^{n-1}}, 0\right)} \Phi\right]\right|=\left|\mathcal{P}_{H} \nu\left(y_{0}\right)\right|=\frac{1}{\sqrt{1+\left|\varpi\left(y_{0}\right)\right|^{2}}} \neq 0 .
$$

We use Theorem 1.3 in the proof of Theorem 1.1, since the nondegeneracy of the Jacobian allows us to locally invert the exponential map $\exp _{S}$.

At this point, we would like to briefly recall some results of a similar flavour in the Euclidean setting. If $N \subset \mathbf{R}^{n}$ is a $\mathbf{C}^{k}$ manifold with $k \geq 2$, then one sees that, near $N$, the distance function $\delta_{N}$ is $\mathbf{C}^{k-1}$-smooth. A first result can be found in the book by Gilbarg and Trudinger [12]. They proved the existence of a neighborhood $U$ of $N$ such that the distance function $\delta_{N}$ is of class $\mathbf{C}^{k}$ on $U \backslash N$. The problem was also considered by Federer for hypersurfaces in the $C^{1,1}$ class; see [8]. The complete solution in the $\mathbf{C}^{1}$ case, can be found in a paper by Krantz and Parks; see [14]. See also Foote [9]. Previous results of this type, in a sub-Riemannian setting, were proved by the first two authors for the first Heisenberg group $\mathbf{H}^{1}$; see [3].

We conclude this introduction by remarking that in our paper we consider "second order" differential objects, which can not be merely reduced to the horizontal directions alone. Indeed, as it has been already pointed out in [3] and [4], while in the Riemannian spaces the notion of metric normal to a smooth hypersurface $S$ in a point $p$ is totally encoded in the normal vector to $S$ at $p$ (see [8]), in sub-Riemannian spaces the intrinsic natural object that could be considered, the so called horizontal 
gradient, is not sufficient to characterize the metric normal. There exist in fact infinitely many different geodesics having the same horizontal initial speed vector leaving a fixed point in a Carnot group, that never coincide except that in the initial point. We covered this lack of information by using the vector $\varpi$, which generalizes the imaginary curvature of $[3,4]$.

Theorem 1.1 was used in [27, pp. 53-55], who cited a much earlier version of this article. Given a compact hypersurface $S$ without characteristic points in the Heisenberg group $\mathbf{H}^{n}$, Theorem 1.1 can be used to construct in the open set $U$ of the points having the unique nearest point property, a foliation $\left\{S_{r}: r \in D\right\}$ (here $D$ is a suitable subset of $\left[0,+\infty[)\right.$ such that $E: \cup_{r \in D} S_{r}$ is Ahlfors regular and has assigned Hausdorff dimension $\alpha \in] 2 n, 2 n+2\left[\right.$. The leaf $S_{r}$ is the hypersurface of the points $p$ in $U$ such that $\delta^{s}(p)=r$.

For different perspectives on sub-Riemannian geometry we refer the reader to $[2,6,11,21,22,26,29]$.

\section{Preliminary results}

In this section we introduce notation and concepts used in the proofs of the main theorems. Most of this material is known and it is presented here with a unified notation for ease of the reader. The basic notions on the sub-Riemannian geometry of Carnot groups, together with a basic vocabulary of hypersurfaces are introduced in Section 2.1.

In Section 2.2 we first define the sub-Riemannian exponential map and, since we are in the 2-step case, we then explicitly integrate the normal geodesic equations. The results in Sections 2.1, 2.2 are necessary to prove Theorem 1.2 and they are then used in the proofs of Theorems 1.1 and 1.3.

2.1. Basics on 2-step Carnot groups and hypersurfaces. Some references concerning sub-Riemannian geometry and Carnot groups can be found, for instance, in $[10,18,17,21,22,26,19]$. In particular we focus the case of 2-step Carnot groups.

Let $(\mathbf{G}, \bullet)$ be a 2 -step Carnot group (with respect to a group law $\bullet$ ). In other words, $\mathbf{G}$ is a $n$-dimensional, connected, simply connected, nilpotent and stratified Lie group and the Lie algebra $\mathfrak{g} \subset \mathfrak{X}(\mathbf{G})$ of all left-invariant vector fields on $\mathbf{G}$ satisfies:

$$
\mathfrak{g}=H \oplus V, \text { where }[H, H]=V \text { and }[H, V]=0 .
$$

The layers $H$ and $V$ of the stratification of $\mathfrak{g}$ are called horizontal and vertical, respectively. We set $h:=\operatorname{dim}(H)$ and $v:=n-h=\operatorname{dim}(V)$. Let 0 denote the identity of $\mathbf{G}$. Recall that any point $x \in \mathbf{G}$ defines a smooth left-translation map $L_{x}: \mathbf{G} \longrightarrow \mathbf{G}$ by $L_{x}(y):=x \bullet y$ for any $y \in \mathbf{G}$. A vector field $X \in \mathfrak{X}(\mathbf{G})$ is called left-invariant if $L_{y_{*}} X(x)=X(y \bullet x)$ for every $x, y \in \mathbf{G}$, where $L_{y_{*}}$ denotes the differential of the left-translation. The Lie algebra $\mathfrak{g}$ of $\mathbf{G}$ is made isomorphic to $T_{0} \mathbf{G}$ by identifying each left-invariant vector field $X$ with its value at 0 , and the isomorphism is given by $L_{x *}: T_{0} \mathbf{G} \longrightarrow T_{x} \mathbf{G}$. Hence, we have $\mathfrak{g} \cong \mathbf{R}^{n}=\mathbf{R}^{h} \oplus \mathbf{R}^{v}$, where $H \cong \mathbf{R}^{h}$ and $V \cong \mathbf{R}^{v}$.

Let us set $\mathcal{I}_{H}:=\{1, \ldots, h\}, \mathcal{I}_{V}:=\{h+1, \ldots, n\}$. We use Latin letters $i, j, k, \ldots$, for indices belonging to $\mathcal{I}_{H}$ and Greek letters $\alpha, \beta, \gamma, \ldots$, for indices belonging to $\mathcal{I}_{V}$. Unless otherwise specified, we use capital Latin letters $I, J, K, \ldots$, for indices from 1 to $n$.

Let $g_{H}=\langle\cdot, \cdot\rangle_{H}$ be a positive definite bilinear form on $H$ : it defines a $s u b$ Riemannian metric on $\mathbf{G}$. Let $\left\{X_{1}, \ldots, X_{h}\right\}$ be a left-invariant orthonormal basis 
for $H$, with respect to $g_{H}$. This basis can be completed to a global basis of leftinvariant vector fields of $\mathbf{G}$, say $\left\{X_{1}, \ldots, X_{n}\right\}$, with $X_{h+1}, \ldots, X_{n}$ in $V$. We also extend $\langle\cdot, \cdot\rangle_{H}$ to a Riemannian metric $g=\langle\cdot, \cdot\rangle$ on $\mathbf{G}$, by requiring that $\left\{X_{1}, \ldots, X_{n}\right\}$ is an orthonormal basis for $\langle\cdot, \cdot\rangle$. This might be done in many ways, but our results are independent of the particular extension. We shall assume that $X_{I}(0)=\mathrm{e}_{I}$ where $\mathrm{e}_{I}:=(0, \ldots, 0, \underbrace{1}_{I-\text { th place }}, 0, \ldots, 0)$ is the $I$-th vector of the canonical basis of $\mathbf{R}^{n} \cong$ $T_{0} \mathbf{G}$ for any $I=1, \ldots, n$. Equivalently, $X_{I}(x)=L_{x *} \mathrm{e}_{I}$ for any $I=1, \ldots, n$.

The structure constants of the Lie algebra $\mathfrak{g}$ associated with the global leftinvariant basis $\left\{X_{1}, \ldots, X_{n}\right\}$ are defined by

$$
C_{I J}^{R}:=\left\langle\left[X_{I}, X_{J}\right], X_{R}\right\rangle \quad \forall I, J, R=1, \ldots, n ;
$$

see [28]. Note that they are skew-symmetric and satisfy Jacobi's identity. In addition, the stratification hypothesis implies that $C_{I J}^{R} \neq 0$ only if $I, J \in \mathcal{I}_{H}$ and $R \in \mathcal{I}_{V}$.

Definition 2.1. Throughout this paper, we will use the following notation:

(i) $C_{H}^{\alpha}:=\left[C_{i j}^{\alpha}\right]_{i, j \in \mathcal{I}_{H}} \in \mathcal{M}_{h \times h}(\mathbf{R}) \quad \forall \alpha \in \mathcal{I}_{V}$

(ii) $C_{H}(Z):=\sum_{\alpha \in \mathcal{I}_{V}} z_{\alpha} C_{H}^{\alpha} \in \mathcal{M}_{h \times h}$,

where $Z=\sum_{\alpha \in \mathcal{I}_{V}} z_{\alpha} X_{\alpha} \in V$.

These matrices can be seen as linear operators acting on $H$. From now on we shall fix exponential coordinates of the 1 st kind on $\mathbf{G}$ (see [28]) so that every $p \in \mathbf{G}$ is represented as an $n$-tuple $x=\left(x_{1}, \ldots, x_{n}\right) \in \mathbf{R}^{n}$, that is $p=\exp _{\mathfrak{g}}\left(\sum_{I} x_{I} \mathrm{e}_{I}\right) \in \mathbf{G}$, where $\exp _{\mathfrak{g}}: \mathfrak{g} \rightarrow \mathbf{G}$ denotes the Lie group exponential map. For the sake of simplicity we shall set

$$
x_{H}:=\left(x_{1}, \ldots, x_{h}\right) \in \mathbf{R}^{h}, \quad x_{V}:=\left(x_{h+1}, \ldots, x_{n}\right) \in \mathbf{R}^{v} .
$$

Hence, we shall sometimes write $p=\exp _{\mathfrak{g}}\left(x_{H}, x_{V}\right)\left(\right.$ and $\left.p \equiv\left(x_{H}, x_{V}\right)\right)$.

Carnot groups are homogeneous groups; see [21]. For the 2-step case, this means that there exists a 1-parameter group of automorphisms $\delta_{t}: \mathbf{G} \rightarrow \mathbf{G}$ for any $t>0$ defined as $\delta_{t} p:=\exp _{\mathfrak{g}}\left(t x_{H}, t^{2} x_{V}\right)$ whenever $p=\exp _{\mathfrak{g}}\left(x_{H}, x_{V}\right) \in \mathbf{G}$.

The Baker-Campbell-Hausdorff formula uniquely determines the group law $\bullet$ of $\mathbf{G}$ from the structure of its Lie algebra $\mathfrak{g}$. In fact, one has

$$
\exp _{\mathfrak{g}}(X) \bullet \exp _{\mathfrak{g}}(Y)=\exp _{\mathfrak{g}}(X \star Y) \quad \forall X, Y \in \mathfrak{g},
$$

where $\star: \mathfrak{g} \times \mathfrak{g} \longrightarrow \mathfrak{g}$ is the Baker-Campbell-Hausdorff product defined by

$$
X \star Y=X+Y+\frac{1}{2}[X, Y]
$$

In exponential coordinates, formula (5) yields a polynomial group law. More precisely, if $p=\exp _{\mathfrak{g}}\left(\sum_{I} x_{I} \mathrm{e}_{I}\right)$ and $q=\exp _{\mathfrak{g}}\left(\sum_{I} y_{I} \mathrm{e}_{I}\right) \in \mathbf{G}$, then

$$
p \bullet q=\exp _{\mathfrak{g}}\left(\sum_{I=1}^{n}\left(x_{I}+y_{I}\right) \mathrm{e}_{I}-\frac{1}{2} \sum_{\alpha \in \mathcal{I}_{V}}\left\langle C_{H}^{\alpha} x_{H}, y_{H}\right\rangle \mathrm{e}_{\alpha}\right),
$$

where $C_{H}^{\alpha}=\left[C_{i j}^{\alpha}\right]_{i, j \in \mathcal{I}_{H}}$. Note that the inverse of any $p=\exp _{\mathfrak{g}}\left(x_{1}, \ldots, x_{n}\right) \in \mathbf{G}$ is given by $p^{-1}=\exp _{\mathfrak{g}}\left(-x_{1}, \ldots,-x_{n}\right)=-p$ and that $0=\exp _{\mathfrak{g}}(0, \ldots, 0) \in \mathbf{G}$ (in particular, one has $\left.0=\exp _{\mathfrak{g}}\left(0_{H}, 0_{V}\right)\right)$. 
The global left-invariant basis $\left\{X_{1}, \ldots, X_{n}\right\}$ can explicitly be written out with respect to the canonical basis $\left\{\mathrm{e}_{1}, \ldots, \mathrm{e}_{n}\right\}$ of $\mathbf{R}^{n}$ as follows:

$$
\begin{aligned}
& X_{i}(x)=L_{x *} \mathrm{e}_{i}=\mathrm{e}_{i}-\frac{1}{2} \sum_{\alpha \in \mathcal{I}_{V}}\left\langle C_{H}^{\alpha} x_{H}, \mathrm{e}_{i}\right\rangle \mathrm{e}_{\alpha} \quad \forall i \in \mathcal{I}_{H} \\
& X_{\alpha}(x)=L_{x *} \mathrm{e}_{\alpha}=\mathrm{e}_{\alpha} \quad \forall \alpha \in \mathcal{I}_{V} .
\end{aligned}
$$

The Carnot-Carathéodory distance d (abbreviated as CC) associated with the subRiemannian structure $g_{H}$ can now be defined, and it makes $(\mathbf{G}, d)$ into a complete geodesic metric space; see [6, 21].

(i) An absolutely continuous curve $\gamma:[0, r] \rightarrow \mathbf{G}$ is called horizontal if, for $\mathcal{L}^{1}$-a.e. $s \in[0, r]$, one has $\gamma^{\prime}(t) \in H_{\gamma(t)}=\operatorname{span}_{\mathbf{R}}\left\{X_{1}(\gamma(t)), \ldots X_{h}(\gamma(t))\right\}$.

(ii) By definition, the length of a horizontal curve $\gamma$ is given by

$$
l_{H}(\gamma):=\int_{0}^{r} \sqrt{\sum_{i \in \mathcal{I}_{H}}\left\langle\gamma^{\prime}(t), X_{i}(\gamma(t))\right\rangle_{H}^{2}} d t .
$$

(iii) If $p, q \in \mathbf{G}$ then, the $C C$-distance between $p$ and $q$ is given by

$$
d(p, q):=\inf \left\{l_{H}(\gamma): \gamma \text { is a horizontal curve connecting } p \text { with } q\right\} .
$$

We shall denote by $B(p, r)$ the open metric ball of center $p \in \mathbf{G}$ and radius $r>0$. Furthermore, we shall set $\mathbf{S}(p, r):=\partial B(p, r)$.

By definition, any absolutely continuous horizontal curve connecting $p$ with $q$, and realizing the minimum $d(p, q)$ is called a length-minimizing geodesic. The existence of horizontal curves connecting each pair of points is ensured by Chow's Theorem; see [21]. Further details about sub-Riemannian geodesics can be found in Section 2.2.

Let $x \in \mathbf{G}$. Throughout this paper, the maps

$$
\mathcal{P}_{H}: T_{x} \mathbf{G} \longrightarrow H_{x}, \quad \mathcal{P}_{V}: T_{x} \mathbf{G} \longrightarrow V_{x}
$$

will denote the orthogonal projection operators onto the vector subspaces $H_{x}$ and $V_{x}$ of $T_{x} \mathbf{G}$, respectively. For any function $f$ of class $\mathbf{C}^{1}$, we set

$$
\begin{aligned}
& \operatorname{grad}_{H} f:=X_{1}(f) X_{1}+\cdots+X_{h}(f) X_{h} \equiv\left(X_{1} f, \ldots, X_{h} f\right), \\
& \operatorname{grad}_{V} f:=X_{h+1}(f) X_{h+1}+\cdots+X_{n}(f) X_{n} \equiv\left(X_{h+1} f, \ldots, X_{n} f\right) .
\end{aligned}
$$

Together, these vectors form a decomposition of the Riemannian gradient, that is, $\operatorname{grad} f=\operatorname{grad}_{H} f+\operatorname{grad}_{V} f$ (or equivalently, $\operatorname{grad}_{H} f(x)=\mathcal{P}_{H}(\operatorname{grad} f(x))$ and $\operatorname{grad}_{V} f(x)=\mathcal{P}_{V}(\operatorname{grad} f(x))$ for any $\left.x \in \mathbf{G}\right)$.

We now recall some basic facts about hypersurfaces. This material can be found, for instance, in [18]. Let $S \subset \mathbf{G}$ be a smooth hypersurface, that is an $(n-1)$ dimensional smooth submanifold of $\mathbf{G}$. We will always assume that $S=\partial \Omega$ is connected and it is the boundary of an open subset $\Omega$ of $\mathbf{G}$. In particular, $S$ is orientable. For non orientable manifolds our results maintain an obvious local character.

Definition 2.2. Let $S \subset \mathbf{G}$ be a hypersurface of class $\mathbf{C}^{k}$ with $k \geq 2$. Let $C_{S}:=\left\{x \in S: H_{x} \subset T_{x} S\right\}$ be the characteristic set of $S$ and let $\nu$ be its Riemannian unit outward normal vector. The unit $H$-normal vector along $S_{0}:=S \backslash C_{S}$ is the normalized projection of $\nu$ onto $H$, that is $\nu_{H}:=\frac{\mathcal{P}_{H} \nu}{\left|\mathcal{P}_{H} \nu\right|}$. We shall set:

(i) $\mathcal{N}:=\frac{\nu}{\left|\mathcal{P}_{H} \nu\right|}=\left(\nu_{H}, \varpi\right)$, where $\varpi:=\frac{\mathcal{P}_{V} \nu}{\left|\mathcal{P}_{H} \nu\right|}$;

(ii) $\varpi_{\alpha}:=\frac{\nu_{\alpha}}{\left|\mathcal{P}_{H} \nu\right|} \quad \forall \alpha \in \mathcal{I}_{V}$;

(iii) $C_{H}(\varpi):=\sum_{\alpha \in \mathcal{I}_{V}} \varpi_{\alpha} C_{H}^{\alpha}$. 
Whenever we need to stress the dependence of these vectors on $S$, we shall simply write $\nu^{S}, \nu_{H}^{S}, \varpi^{S}, \mathcal{N}^{S}$. Here we just remark that the $\mathbf{C}^{2}$ regularity assumption on $S$ ensures that the Riemannian measure of the characteristic set $C_{S}$ vanishes. Precise estimates of the size of $C_{S}$ can be found in [5].

Finally, let

$$
\delta: \mathbf{G} \longrightarrow \mathbf{R}^{+} \cup\{0\}
$$

denote the $C C$-distance function from $S$, that is $\delta(x):=\inf _{y \in S} d(x, y)$. For the sake of simplicity, we can always think of $S$ as the zero set of a (smooth) function $f: \mathbf{G} \rightarrow \mathbf{R}$ with nonvanishing gradient on $S$, that is $S=\{f=0\}=\partial \Omega$ (where $\Omega=\{f<0\}$ ). Fix $x_{0} \in S_{0}$ and let $B\left(x_{0}, r\right)$ be a CC-ball such that $B\left(x_{0}, r\right) \cap S=B\left(x_{0}, r\right) \cap S_{0}$. Without loss of generality, we assume that $\nu_{H}(x)=\frac{\operatorname{grad}_{H} f(x)}{\left|\operatorname{grad}_{H} f(x)\right|}$ at any smooth point $x \in B\left(x_{0}, r\right) \cap S=B\left(x_{0}, r\right) \cap\{f=0\}$. In this way, we can now localize the upper portion of $B\left(x_{0}, r\right)$ with respect to $S$, by setting $B\left(x_{0}, r\right)^{+}:=B\left(x_{0}, r\right) \backslash \Omega$. Hence, we can define the signed distance function to $S$ as

$$
\delta^{s}(x):= \begin{cases}\delta(x) & \text { if } x \in B\left(x_{0}, r\right)^{+} \\ -\delta(x) & \text { if } x \in B\left(x_{0}, r\right) \backslash B\left(x_{0}, r\right)^{+} .\end{cases}
$$

We would like to stress that if $S=\mathbf{S}\left(x_{0}, r\right)=\left\{x \in \mathbf{G}: d\left(x, x_{0}\right)=r\right\}$, then at any smooth point of $S$ the eikonal equation (that is, $\left|\operatorname{grad}_{H} d\right|=1 \mathcal{L}^{n}$-a.e.; see [20]) implies that $\nu_{H}^{\mathbf{S}\left(x_{0}, r\right)}=\operatorname{grad}_{H} d$ and that $\varpi^{\mathbf{S}\left(x_{0}, r\right)}=\operatorname{grad}_{V} d$, where $d$ denotes the CC-distance function to $x_{0}$, that is $\mathbf{G} \ni x \mapsto d\left(x, x_{0}\right)$. In particular, note that any smooth point of the CC-sphere $\mathbf{S}\left(x_{0}, r\right)$ is necessarily non characteristic.

2.2. Sub-Riemannian exponential map and integration of the normal geodesic equations. The main references for this section are [2, 24, 25, 29]. Starting from the system (1) for normal geodesics, by the standard ODEs theory we obtain existence, uniqueness, regularity and smooth dependence on the initial data for small times. Fix $x_{0} \in \mathbf{G}$. We shall denote by

$$
\exp _{\mathcal{S R}}\left(x_{0}, v_{0}\right)(\cdot):\left[0,+\infty\left[\longrightarrow \mathbf{G}, \quad \exp _{\mathcal{S R}}\left(x_{0}, v_{0}\right)(t) \quad \forall t>0,\right.\right.
$$

the unique normal geodesic starting from $x_{0}$ with initial condition $v(0)=v_{0}$ for some fixed vector $v_{0}=v_{H}(0)+v_{V}(0) \in H_{x_{0}} \oplus V_{x_{0}}$.

In order to define a sub-Riemannian equivalent to the exponential map we state the following straightforward result whose proof we skip.

Lemma 2.3. (Homogeneity) The identity $\exp _{\mathcal{S R}}\left(x_{0}, a v_{0}\right)(t)=\exp _{\mathcal{S R}}\left(x_{0}, v_{0}\right)(a t)$ holds for any $x_{0} \in \mathbf{G}, v_{0} \in T_{x_{0}} \mathbf{G}, t \geq 0, a>0$.

Definition 2.4. (Sub-Riemannian exponential map) We shall set

$$
\exp _{\mathcal{S R}}\left(x_{0}, \cdot\right): T_{x_{0}} \mathbf{G} \longrightarrow \mathbf{G}, \quad \exp _{\mathcal{S R}}\left(x_{0}, v_{0}\right)=\exp _{\mathcal{S R}}\left(x_{0}, v_{0}\right)(1) \text {. }
$$

The map $\exp _{\mathcal{S R}}\left(x_{0}, \cdot\right)$ is the sub-Riemannian exponential map at $x_{0} \in \mathbf{G}$.

The map $\exp _{\mathcal{S R}}\left(x_{0}, \cdot\right)$ plays in sub-Riemannian geometry a role similar to that of the ordinary exponential map in Riemannian geometry. But there are many differences and its structure is much more complicated. An important difference is that $\exp _{\mathcal{S R}}\left(x_{0}, \cdot\right)$ is not a diffeomorphism on any neighborhood of the origin in $H_{x_{0}} \oplus V_{x_{0}}$, as it is clear from the Heisenberg group, the simplest non-trivial example of Carnot group; see [21, 29].

Since we will need a description of the sub-Riemannian exponential map, we now perform an explicit integration of the ODEs system for normal geodesics. To begin 
with, let $x:[0, r[\subset \mathbf{R} \longrightarrow \mathbf{G}$ be a normal geodesic (for some $r>0$ ). From the system (1), we see that $v_{V}(t) \in V$ is independent of $t \in[0, r$. Later on we shall set $v_{V} \equiv v_{V}(t)=v_{V}(0)$. By the standard ODEs theory, we have that $v_{H}(t) \in H$ is given by

$$
v_{H}(t)=e^{-C_{H}\left(v_{V}\right) t} v_{H}(0) \quad \forall t \in[0, r[.
$$

We also remark that the equation $x^{\prime}=v_{H}$ is equivalent to the following scalar equations:

$$
\begin{cases}x_{i}^{\prime}=v_{i} & \forall i \in \mathcal{I}_{H}, \\ x_{\alpha}^{\prime}=-\frac{1}{2}\left\langle C_{H}^{\alpha} x_{H}, v_{H}\right\rangle, & \forall \alpha \in \mathcal{I}_{V} .\end{cases}
$$

Now, let us set

$$
x_{H}(t):=\left(x_{1}(t), \ldots, x_{h}(t)\right) \in \mathbf{R}^{h} \cong H, \quad x_{V}(t):=\left(x_{h+1}(t), \ldots, x_{n}(t)\right) \in \mathbf{R}^{v} \cong V ;
$$

see (4) in Section 2.1. Thus, we get

$$
x_{H}(t)=x_{H}(0)+\int_{0}^{t} e^{-C_{H}\left(v_{V}\right) s} v_{H}^{0} d s .
$$

Moreover, since $x_{\alpha}(t)=x_{\alpha}(0)-\frac{1}{2} \int_{0}^{t}\left\langle C_{H}^{\alpha} x_{H}(s), x_{H}^{\prime}(s)\right\rangle d s$, we have

$$
x_{V}(t)=\sum_{\alpha \in \mathcal{I}_{V}}\left(x_{\alpha}(0)-\frac{1}{2} \int_{0}^{t}\left\langle C_{H}^{\alpha} x_{H}(s), x_{H}^{\prime}(s)\right\rangle d s\right) \mathrm{e}_{\alpha} .
$$

The equations (9) and (10) together describe the sub-Riemannian exponential map in the 2-step Carnot group case. More precisely, after fixing a base point $x_{0}=$ $\exp _{\mathfrak{g}}\left(x_{H}^{0}, x_{V}^{0}\right) \in \mathbf{G}$, we have

$$
\exp _{\mathcal{S R}}\left(x_{0}, v_{0}\right)(t):=\exp _{\mathfrak{g}}\left(x_{H}(t), x_{V}(t)\right) \quad \forall t \in[0, r[
$$

and hence $\exp _{\mathcal{S R}}\left(x_{0}, v_{0}\right)=\exp _{\mathfrak{g}}\left(x_{H}(1), x_{V}(1)\right)$; see Definition 2.4. As already said in Section 2.1, we are identifying the group $\mathbf{G}$ with its Lie algebra, so that any curve in $\mathrm{G}$ might be seen as a curve in $\mathfrak{g}$. More precisely, one has

$$
\begin{aligned}
\exp _{\mathcal{S R}}\left(x_{0}, v_{0}\right)(t) \equiv & \left(x_{H}(t), x_{V}(t)\right)=(\underbrace{\left(x_{H}^{0}+\int_{0}^{t} e^{-C_{H}\left(v_{V}\right) s} v_{H}^{0} d s\right)}_{=x_{H}(t)}, \\
& \left.\left(x_{V}^{0}-\frac{1}{2} \sum_{\alpha \in \mathcal{I}_{V}}\left(\int_{0}^{t}\left\langle C_{H}^{\alpha} x_{H}(s), x_{H}^{\prime}(s)\right\rangle d s\right) \mathrm{e}_{\alpha}\right)\right) .
\end{aligned}
$$

Remark 2.5. For unit speed normal geodesics the map $\exp _{\mathcal{S R}}\left(x_{0}, \cdot\right)(\cdot)$ can be defined by using the hypercylinder $U H \times V$, where $U H:=\{X \in H:|X|=1\}$ denotes the set of all unit horizontal vectors, which is isomorphic to the unit Euclidean sphere $\mathbf{S}^{h-1} \subset \mathbf{R}^{h}$, that is, $U H \cong \mathbf{S}^{h-1}$. In other words, we can assume that $\exp _{\mathcal{S R}}\left(x_{0}, \cdot\right)(\cdot): U H \times V \times \mathbf{R} \longrightarrow \mathbf{G}$.

\section{Geometric lemmas}

3.1. Proof of Theorem 1.2. Let $\mathbf{S}\left(x_{0}, t\right)=\left\{x \in \mathbf{G}: d\left(x, x_{0}\right)=t\right\}$ be the CCsphere, with $x_{0} \in \mathbf{G}$ and $t>0$. As already said, at any smooth point of $\mathbf{S}\left(x_{0}, t\right)$ the eikonal equation implies that $\nu_{H} \equiv \nu_{H}^{\mathbf{S}\left(x_{0}, t\right)}=\operatorname{grad}_{H} d$ and that $\varpi \equiv \varpi^{\mathbf{S}\left(x_{0}, t\right)}=\operatorname{grad}_{V} d$, where $d(x):=d\left(x_{0}, x\right)$ represents the CC-distance function to $x_{0}$; see Definition 2.2. 
We preliminarily observe that Theorem 1.2 can be restated as follows: assuming that $\nu_{H} \equiv \nu_{H}^{\mathbf{S}\left(x_{0}, t\right)}$ and $\varpi \equiv \varpi^{\mathbf{S}\left(x_{0}, t\right)}$ at any smooth point of $\mathbf{S}\left(x_{0}, t\right)$, then (i) is equivalent to the vector equation:

$$
\frac{d}{d t} x(t)=\nu_{H}(x(t))
$$

moreover, (ii) is equivalent to the following two vector equations:

$$
\begin{aligned}
\frac{d}{d t} \nu_{H}(x(t)) & =-C_{H}(\varpi(x(t))) \nu_{H}(x(t)), \\
\frac{d}{d t} \varpi(x(t)) & =0 ;
\end{aligned}
$$

see Definitions 2.2 and 2.1 .

Let us prove this statement for the 2-step case: we note, however, that the same argument can easily be extended to $k$-step Carnot groups in general.

Proof of Theorem 1.2. Let $\mathbf{G}$ be a 2-step Carnot group. The calculation below is mainly based on the eikonal equation, first proven in [20]. Concerning the case of the Heisenberg group see also [19]. We start by the system (1), which is satisfied by unit speed normal geodesics. Let us fix the initial conditions: $x(0)=x_{0}, v(0)=v_{0}$, where $v_{0}=v_{H}^{0}+v_{V}^{0},\left|v_{H}^{0}\right|=1$, and set $d(x):=d\left(x, x_{0}\right)$. In particular, we have $d(x(t))=t$ for every $t \in[0, r$ [ (with $r>0$ small enough). By differentiating this identity, we obtain

$$
1=\frac{d}{d t} d(x(t))=\left\langle\operatorname{grad} d(x(t)), x^{\prime}(t)\right\rangle=\left\langle\operatorname{grad}_{H} d(x(t)), x^{\prime}(t)\right\rangle .
$$

Since $x^{\prime}=v_{H}$ and $\left|v_{H}\right|=\left|v_{H}^{0}\right|=1$, the eikonal equation implies that

$$
x^{\prime}(t)=\operatorname{grad}_{H} d(x(t))
$$

for every (sufficiently small) $t>0$, that is (i) in Theorem 1.2. This can be written in coordinates as follows:

$$
x_{i}^{\prime}(t)=X_{i} d(x(t)) \quad \forall i \in \mathcal{I}_{H} .
$$

Let now $I \in\{1, \ldots, n\}$ and let us differentiate the function $X_{I} d$ along the unit speed normal geodesic $x(t)$ defined by the previous assumptions. For the sake of simplicity, in the following computations we shall drop the dependence on the variable $t$. Thus, we have

$$
\begin{aligned}
\frac{d}{d t} X_{I} d(x) & =\left\langle\operatorname{grad}\left(X_{I} d\right)(x), x^{\prime}\right\rangle=\left\langle\operatorname{grad}_{H}\left(X_{I} d\right)(x), x^{\prime}\right\rangle \\
& =\left\langle\operatorname{grad}_{H}\left(X_{I} d\right)(x), \operatorname{grad}_{H}(d)(x)\right\rangle=\sum_{i \in \mathcal{I}_{H}} X_{i} d(x) X_{i}\left(X_{I} d\right)(x) .
\end{aligned}
$$

Note that $X_{i} X_{I}=X_{I} X_{i}+\left[X_{i}, X_{I}\right]=X_{I} X_{i}+\sum_{\beta \in I_{\mathrm{ord}(I)+1}} C_{i I}^{\beta} X_{\beta}$ (in fact, by definition, one has $C_{I J}^{R}=\left\langle\left[X_{I}, X_{J}\right], X_{R}\right\rangle$ and $\left[X_{I}, X_{J}\right]=\sum_{R} C_{I J}^{R} X_{R}$; see Section 2.1. Recall also that, in the 2-step case, we have $C_{I J}^{R} \neq 0$ only if $I, J \in \mathcal{I}_{H}$ and $R \in \mathcal{I}_{V}$. Below, we shall put

$$
\operatorname{ord}(I):= \begin{cases}1 & \text { if } I \in \mathcal{I}_{H} \\ 2 & \text { if } I \in \mathcal{I}_{V}\end{cases}
$$


and set the default value ord $(I)=3$ if $X_{I} \in[H, V]=0$. We have

$$
\begin{aligned}
\frac{d}{d t} X_{I} d(x) & =\sum_{i \in \mathcal{I}_{H}}\left(X_{i} d(x) X_{I}\left(X_{i} d\right)(x)+\sum_{\beta \geq \operatorname{ord}(I)+1} X_{i} d(x) C_{i I}^{\beta} X_{\beta} d(x)\right) \\
& =\frac{1}{2} X_{I}\left(\left|\operatorname{grad}_{H}(d)(x)\right|^{2}\right)+\sum_{i \in \mathcal{I}_{H}} \sum_{\beta \geq \operatorname{ord}(I)+1} X_{i} d(x) C_{i I}^{\beta} X_{\beta} d(x) .
\end{aligned}
$$

The first term in the sum is zero because $\left|\operatorname{grad}_{H} d(x)\right|=1$, by the eikonal equation. Using the skew-symmetry of the structure constants, yields

$$
\frac{d}{d t} X_{I} d(x)=-\sum_{i \in \mathcal{I}_{H}} \sum_{\beta \geq \operatorname{ord}(I)+1} X_{i} d(x) C_{I i}^{\beta} X_{\beta} d(x) .
$$

Now if $I \in \mathcal{I}_{H}$, then $\beta \in \mathcal{I}_{V}$ and (13) becomes

$$
\frac{d}{d t} X_{I} d(x)=-\sum_{i \in \mathcal{I}_{H}}\left(\sum_{\beta \in \mathcal{I}_{V}} C_{I i}^{\beta} X_{\beta} d(x)\right) X_{i} d(x) .
$$

Therefore using (14) and Definition 2.1 yields

$$
\frac{d}{d t} \operatorname{grad}_{H} d(x)=-C_{H}(\varpi) \operatorname{grad}_{H} d(x) .
$$

On the other hand if $I \in \mathcal{I}_{V}$, then $\frac{d}{d t} X_{I} d(x)=0$ and we get that

$$
\frac{d}{d t} \operatorname{grad}_{V} d(x)=0 \text {. }
$$

Equations (15) and (16) prove (ii) in Theorem 1.2.

3.2. Jacobian of the exponential map in 2-step groups. We refer the reader to Sections 2.1 and 2.2 for details on the notation used here. Let $\Omega \subset \mathbf{G}$ be a connected open set and let $S=\partial \Omega$ be a smooth hypersurface in a special ${ }^{1} 2$-step Carnot group G. For any point $x$ belonging to the non characteristic set $S_{0}=S \backslash C_{S}$ of $S$, the notion of unit $H$-normal vector $\nu_{H}(x)$ to $S$ at $x$ was defined in Section 2.1. Unfortunately, there are infinitely many geodesics starting at $x$ and having initial velocity $\nu_{H}(x)$ : only one $\gamma$ among them has the property that $\delta(\gamma(t))=|t|$, at least for small $|t|$. We will see during the proof of Theorem 1.1 that there exists only one geodesic $\gamma$ with this property. We shall call this distinguished $\gamma$ the metric normal to $S$ at $x$. Such notion was first investigated in [3], when $\mathbf{G}=\mathbf{H}^{1}$ denotes the 1st Heisenberg group.

Note that the (non-unit) normal vector $\mathcal{N}$ is defined at non characteristic points only. We also remark that the choice of the sign of $\mathcal{N}$ at a point, hence on the whole of $S$, corresponds to an orientation for $S$ or, which is the same, to the choice of one of the two components $\Omega$ of $\mathbf{G} \backslash S$ such that $S=\partial \Omega$; see Section 2.1.

Remark 3.1. Theorem 1.2 solves the problem of selecting, for any point $x_{1}$ in $\mathbf{S}\left(x_{0}, r\right)$ where the sphere is smooth, the unique unique speed normal geodesic $\gamma(t)$ having initial velocity equal to the unit $H$-normal vector at that point, that is $\gamma^{\prime}(0)=\nu_{H}\left(x_{1}\right)$, and connecting $x_{1}$ to the center $x_{0}$ of $\mathbf{S}\left(x_{0}, r\right)$. Actually, Theorem 1.2 implies that the desired curve must be given by

$$
\gamma(t)=\exp _{\mathcal{S R}}\left(x_{1},-\mathcal{N}\left(x_{1}\right)\right)(t), \quad t \in[0, r],
$$

\footnotetext{
${ }^{1}$ We stress that, in the proof of Theorem 1.3, we do not need this hypothesis.
} 
which, in particular, satisfies $\gamma(0)=x_{1}$ and $\gamma(r)=x_{0}$.

The previous choice of the sign for $\mathcal{N}$ is due to the conventional choice $\Omega=$ $\mathbf{G} \backslash B\left(x_{0}, r\right)$ and reflects the fact that geodesics start on the sphere's boundary and move towards the center as time increases.

As a consequence, Theorem 1.2 admits the following corollary:

Corollary 3.2. Let $S=\{f=0\}$ be the boundary of the set $\Omega=\{f<0\}$, where $f: \mathbf{G} \rightarrow \mathbf{R}$ is a $\mathbf{C}^{2}$ function with nonvanishing gradient. Let $B(y, r)$ denote the $C C$-ball with center $y \in \mathbf{G}$ and radius $r>0$ and assume that $B(y, r) \subset\{f>0\}$ and $\partial B(y, r) \cap S=\{x\}$, where $x \in S_{0}=S \backslash C_{S}$ is non characteristic. Then there exists the metric normal $\gamma_{\mathcal{N}}$ to $S$ at $x$ and one has $\gamma(t) \in \gamma_{\mathcal{N}}$ for every $t \in[0, r]$, where

$$
\gamma(t):=\exp _{\mathcal{S R}}(x, \mathcal{N}(x))(t), \quad t \in[0, r] .
$$

Notice that $\gamma_{\mathcal{N}}$ is a set, while $\gamma$ is a geodesic. We also remind the reader that $\mathcal{N}=\frac{\nu}{\left|\mathcal{P}_{H} \nu\right|}=\left(\nu_{H}, \varpi\right)$, where $\nu$ is the Riemannian unit normal to $S$.

Let $S$ be of class $\mathbf{C}^{k}$ with $k \geq 2$ and define the map

$$
\exp _{S}: S_{0} \times \mathbf{R} \longrightarrow \mathbf{G}, \quad \exp _{S}(y, t):=\exp _{\mathcal{S R}}(y, \mathcal{N}(y))(t),
$$

where $S_{0}=S \backslash C_{S}$ and $\exp _{\mathcal{S R}}$ is the map defined by (7); see Section 2.2 .

The proof of Theorem 1.1 is based on the sub-Riemannian Gauss' Lemma (see Theorem 1.2) and on Theorem 1.3 which will enable us to invert locally the map $\Phi$, obtained from $\exp _{S}$ by using, in a neighborhood of a point $y_{0} \in S_{0}$, a system of Riemannian normal coordinates. More precisely, let $\left(\tau_{1}, \ldots, \tau_{n-1}\right)$ be an orthonormal basis of $T_{y_{0}} S$; for $1 \leq j \leq n-1$ define a real-valued function $u_{j}$ on a neighborhood of $y_{0}$ by setting

$$
u_{j}\left(\left(\exp _{\mathcal{R}}\right)_{y_{0}}\left(\sum_{i=1}^{n-1} t_{i} \tau_{i}\right)\right)=t_{j}
$$

where $\exp _{\mathcal{R}}$ denotes the ordinary Riemannian exponential map. Then, by definition, $u \equiv\left(u_{1}, \ldots, u_{n-1}\right)$ is a system of normal coordinates corresponding to the orthonormal basis $\left(\tau_{1}, \ldots, \tau_{n-1}\right)$; for further details, see [7].

Thus, if $y(u) \equiv y\left(u_{1}, \ldots, u_{n-1}\right)$ describes a neighborhood $U_{0} \subset S_{0}=S \backslash C_{S}$ of a non characteristic point $y_{0} \in S_{0}$, we henceforth set

$$
\Phi(u, t) \equiv \Phi\left(u_{1}, u_{2} \ldots, u_{n-1}, t\right):=\exp _{S}(y(u), t)
$$

where $\left.\Phi: \widetilde{U_{0}} \times\right]-\epsilon, \epsilon\left[\rightarrow \mathbf{G}\right.$ and $\widetilde{U_{0}}$ denotes an open neighborhood of $0_{\mathbf{R}^{n-1}}$ in $\mathbf{R}^{n-1}$. Note that $\Phi\left(0_{\mathbf{R}^{n-1}}, 0\right)=\exp _{S}\left(y_{0}, 0\right)=y_{0}$. Let now $\frac{\partial}{\partial u_{1}}, \ldots, \frac{\partial}{\partial u_{n-1}}$ be the coordinate vector fields associated with our normal coordinate system $\left(u_{1}, \ldots, u_{n-1}\right)$. Then $\mathcal{V}(y):= \pm \frac{\partial y}{\partial u_{1}} \wedge \ldots \wedge \frac{\partial y}{\partial u_{n-1}}$ turns out to be a (possibly non-unit) Riemannian normal vector to $S$ in the neighborhood $U_{0}$ of $y_{0} \in S_{0}$. By construction, one has $\left|\mathcal{V}\left(y_{0}\right)\right|=1$. The Riemannian unit normal $\nu$ to $S$ (in this neighborhood of $y_{0}$ ) is given, up to the sign, by $\nu=\frac{\mathcal{V}}{|\mathcal{V}|}$, while the horizontal unit normal $\nu_{H}$ can be written out as $\nu_{H}=\frac{\mathcal{P}_{H} \mathcal{V}}{\left|\mathcal{P}_{H} \mathcal{V}\right|}$.

Let $\mathcal{J}_{\left(0_{\mathbf{R}^{n-1}}, 0\right)} \Phi$ denote the Jacobian matrix at $\left.\left(0_{\mathbf{R}^{n-1}}, 0\right) \in \widetilde{U_{0}} \times\right]-\epsilon, \epsilon[$.

Proof of Theorem 1.3. Recall that for any base point $x_{0} \in \mathbf{G}$ the map

$$
\exp _{\mathcal{S R}}\left(x_{0}, \cdot\right)(\cdot): U H \times V \times \mathbf{R} \longrightarrow \mathbf{G}
$$


is explicitly given by

$$
\begin{aligned}
& \exp _{\mathcal{S R}}\left(x_{0}, v_{0}\right)(t) \equiv\left(x_{H}(t), x_{V}(t)\right) \\
& =\left(\left(x_{H}^{0}+\int_{0}^{t} e^{-C_{H}\left(v_{V}\right) s} v_{H}^{0} d s\right),\left(x_{V}^{0}-\frac{1}{2} \sum_{\alpha \in \mathcal{I}_{V}}\left(\int_{0}^{t}\left\langle C_{H}^{\alpha} x_{H}(s), x_{H}^{\prime}(s)\right\rangle d s\right) \mathrm{e}_{\alpha}\right)\right)
\end{aligned}
$$

see formula (11) in Section 2.2. Later on we shall set $\Phi=\exp _{\mathfrak{g}}\left(\left(\Phi_{H}, \Phi_{V}\right)\right)$. Thus, assuming $x_{0}=y(u), v_{H}^{0}=\nu_{H}(y(u)), v_{V}^{0}=v_{V}=\varpi(y(u))$, yields

$$
\begin{aligned}
\Phi_{H}(u, t) & =y_{H}(u)+\int_{0}^{t} e^{-C_{H}(\varpi(y(u))) s} \nu_{H}(y(u)) d s \\
& =(\ldots, \underbrace{y_{i}(u)+\left\langle\left(\int_{0}^{t} e^{-C_{H}(\varpi(y(u)) s} \nu_{H}(y(u)) d s\right), \mathrm{e}_{i}\right\rangle}_{i \text { th place }}, \ldots),
\end{aligned}
$$

which can be seen as a vector in $\mathbf{R}^{h} \oplus 0_{\mathbf{R}^{v}}$. Furthermore, we get that

$$
\Phi_{V}(u, t)=y_{V}-\frac{1}{2} \sum_{\alpha \in \mathcal{I}_{V}}\left(\int_{0}^{t}\left\langle C_{H}^{\alpha} \Phi_{H}(u, s), \Phi_{H}^{\prime}(u, s)\right\rangle d s\right) \mathrm{e}_{\alpha}
$$

(where $\left.\Phi_{H}^{\prime}(u, s)=\frac{d}{d s} \Phi_{H}(u, s)\right)$. Therefore, we have

$$
\begin{aligned}
& \Phi(u, t) \\
& \equiv y(u)+\underbrace{\int_{0}^{t} e^{-C_{H}(\varpi(y(u)) s} \nu_{H}(y(u)) d s-\frac{1}{2} \sum_{\alpha \in \mathcal{I}_{V}}\left(\int_{0}^{t}\left\langle C_{H}^{\alpha} \Phi_{H}(u, s), \Phi_{H}^{\prime}(u, s)\right\rangle d s\right) \mathrm{e}_{\alpha}}_{=: \mathcal{A}(y(u), t)} .
\end{aligned}
$$

Now, let us set $\Phi(u, t):=\exp _{\mathfrak{g}}(y(u)+\mathcal{A}(y(u), t))$. Below, for simplicity, we shall often drop the dependence on the variables. By using the explicit expression of $\Phi$ together with the Fundamental Theorem of Calculus, we easily get

$$
\frac{\partial \Phi_{H}}{\partial t}(u, t)=e^{-C_{H}(\varpi(y(u))) t} \nu_{H}(y(u)), \quad \frac{\partial \Phi_{V}}{\partial t}(u, t)=\frac{1}{2} \sum_{\alpha \in \mathcal{I}_{V}}\left\langle C_{H}^{\alpha} \Phi_{H}^{\prime}(u, t), \Phi_{H}(u, t)\right\rangle \mathrm{e}_{\alpha},
$$

where we have applied the skew-symmetry property of the structure constants matrices $C_{H}^{\alpha}$ 's $\left(\alpha \in \mathcal{I}_{V}\right)$. From the previous calculations we obtain

$$
\left(\left.\frac{\partial \Phi}{\partial t}\right|_{t=0}\right)^{T r} \equiv \sum_{i \in \mathcal{I}_{H}} \nu_{H}^{i}(y(u)) \mathrm{e}_{i}+\frac{1}{2} \sum_{\alpha \in \mathcal{I}_{V}}\left\langle C_{H}^{\alpha} \nu_{H}(y(u)), y_{H}(u)\right\rangle \mathrm{e}_{\alpha}=\nu_{H}(y(u)),
$$

where we have used that

$$
\nu_{H}(y)=\sum_{i \in \mathcal{I}_{H}} \nu_{H}^{i}(y) X_{i}(y)=\sum_{i \in \mathcal{I}_{H}} \nu_{H}^{i}(y) \underbrace{\left(\mathrm{e}_{i}-\frac{1}{2} \sum_{\alpha \in \mathcal{I}_{V}}\left\langle C_{H}^{\alpha} y, \mathrm{e}_{i}\right\rangle \mathrm{e}_{\alpha}\right)}_{=X_{i}(y)} .
$$

Differentiating $\Phi$ with respect to the local coordinates $\left(u_{1}, \ldots, u_{n-1}\right)$ yields

$$
\frac{\partial \Phi}{\partial u_{i}} \equiv \frac{\partial y}{\partial u_{i}}+\frac{\partial \mathcal{A}}{\partial u_{i}} \quad \forall i=1, \ldots, n-1 .
$$


Setting $\mathcal{A}=\left(\mathcal{A}_{H}, \mathcal{A}_{V}\right)$, it turns out that

$$
\begin{aligned}
\frac{\partial \mathcal{A}_{H}}{\partial u_{i}} & =\int_{0}^{t}\left(\left[\mathcal{J}_{y}\left(e^{-C_{H}(\varpi(y)) s} \nu_{H}(y)\right)\right] \frac{\partial y}{\partial u_{i}}\right) d s \in \mathbf{R}^{h} \oplus 0_{\mathbf{R}^{v}} \\
\frac{\partial \mathcal{A}_{V}}{\partial u_{i}} & =-\frac{1}{2} \sum_{\alpha \in \mathcal{I}_{V}}\left(\int_{0}^{t}\left\langle\operatorname{grad}_{y}\left(\left\langle C_{H}^{\alpha} \Phi_{H}(u, s), \Phi_{H}^{\prime}(u, s)\right\rangle\right), \frac{\partial y}{\partial u_{i}}\right\rangle d s\right) \mathrm{e}_{\alpha} \in 0_{\mathbf{R}^{h}} \oplus \mathbf{R}^{v}
\end{aligned}
$$

for any $i=1, \ldots, n-1$. Therefore, choosing $t=0$, one gets

$$
\left.\mathcal{J}_{(u, t)} \Phi(u, t)\right|_{(u, 0)}=\operatorname{col}\left[\frac{\partial y}{\partial u_{1}}, \ldots, \frac{\partial y}{\partial u_{n-1}},\left.\frac{\partial \Phi}{\partial t}\right|_{t=0}\right]=\operatorname{col}\left[\frac{\partial y}{\partial u_{1}}, \ldots, \frac{\partial y}{\partial u_{n-1}}, \nu_{H}(y)\right]
$$

because $\left.\frac{\partial \mathcal{A}_{H}}{\partial u_{i}}\right|_{t=0}=0_{\mathbf{R}^{n}}$ and $\left.\frac{\partial \mathcal{A}_{V}}{\partial u_{i}}\right|_{t=0}=0_{\mathbf{R}^{n}}$. Notice that this result it is not a consequence of a chain rule, but it follows from the integral representation of $\mathcal{A}$.

We may now compute the Jacobian determinant of $\mathcal{J}_{(u, 0)} \Phi$. By standard Linear Algebra arguments we have

$$
\begin{aligned}
\left|\operatorname{det}\left[\mathcal{J}_{(u, 0)} \Phi\right]\right| & =\left|\operatorname{det}\left(\operatorname{col}\left[\frac{\partial y}{\partial u_{1}}, \ldots, \frac{\partial y}{\partial u_{n-1}}, \nu_{H}(y)\right]\right)\right| \\
& =\left|\left\langle\left(\frac{\partial y}{\partial u_{1}} \wedge \ldots \wedge \frac{\partial y}{\partial u_{n-1}}\right), \nu_{H}(y)\right\rangle\right| \\
& =\left|\frac{\partial y}{\partial u_{1}} \wedge \ldots \wedge \frac{\partial y}{\partial u_{n-1}}\right|\left|\left\langle\nu(y), \nu_{H}(y)\right\rangle\right| \\
& =\left|\frac{\partial y}{\partial u_{1}} \wedge \ldots \wedge \frac{\partial y}{\partial u_{n-1}}\right|\left|\mathcal{P}_{H} \nu(y)\right|=\left|\mathcal{P}_{H} \mathcal{V}(y)\right| .
\end{aligned}
$$

Finally, observe that at $y_{0}$ we have $\mathcal{V}\left(y_{0}\right)=\nu\left(y_{0}\right)$. This in turn implies that $\left|\operatorname{det}\left[\mathcal{J}_{\left(0_{\mathbf{R}^{n-1}}, 0\right)} \Phi\right]\right|=\left|\mathcal{P}_{H} \nu\left(y_{0}\right)\right|=\frac{1}{\sqrt{1+\left|\varpi\left(y_{0}\right)\right|^{2}}}$, as wished.

As usual, let us set $S_{0}=S \backslash C_{S}$ and observe that $S_{0}$ is an open subset of $S$, in the relative topology. Moreover, since we are assuming that $S$ is of class $\mathbf{C}^{k}$ with $k \geq 2$, one has $\operatorname{dim}_{\text {Riem-Hau }} C_{S} \leq(n-2)$; see [5]. Now let $U_{0} \Subset S_{0}$ be an open set compactly contained in $S_{0}$. For the sake of simplicity, we identify $U_{0}$ with an open neighborhood $\widetilde{U_{0}}$ of $0_{\mathbf{R}^{n-1}}$ in $\mathbf{R}^{n-1}$ by means of a normal coordinate patch; hence, we identify the map $\Phi$ with the exponential $\exp _{S}$, exactly as in (18). By Theorem 1.3 we know that the Jacobian of the mapping $\left.\Phi: \widetilde{U_{0}} \times\right]-\epsilon, \epsilon[\longrightarrow \mathbf{G}$ is non-zero along $\widetilde{U_{0}} \times\{0\}$. Thus, the Inverse Mapping Theorem easily implies the following:

Corollary 3.3. (Invertibility at $S_{0}$ ) There exists $\left.\epsilon_{0} \in\right] 0, \epsilon[$ such that

$$
\left.\exp _{S}: U_{0} \times\right]-\epsilon_{0}, \epsilon_{0}\left[\longrightarrow \exp _{S}\left(U_{0} \times\right]-\epsilon_{0}, \epsilon_{0}[)\right.
$$

is a diffeomorphism of class $\mathbf{C}^{k-1}$.

Notation 3.4. (Projection mapping) Corollary 3.3 enables us to define the following mapping:

$$
\Psi:=\exp _{S}^{-1}: \exp _{S}\left(U_{0} \times\right]-\epsilon_{0}, \epsilon_{0}[) \longrightarrow \underbrace{\left.U_{0} \times\right]-\epsilon_{0}, \epsilon_{0}}_{=: W_{0}}[.
$$

By construction, $\Psi$ is of class $\mathbf{C}^{k-1}$. In the sequel, we shall denote by $\Psi_{S}$ the projection of the map $\Psi$ onto its 1st factor, that is $\Psi(x)=\left(\Psi_{S}(x), t(x)\right)$. 


\section{Proof of Theorem 1.1}

Proof of Theorem 1.1. Let $p_{0}$ be a point of $S_{0}=S \backslash C_{S}$ and consider the exponential map $\exp _{S}: S_{0} \times \mathbf{R} \rightarrow \mathbf{G}$ defined by (17). Then, by Corollary 3.3, there is an open subset $U_{0}$ of $S_{0}:=S \backslash C_{S}$ containing $p_{0}$, such that $\left.\exp _{S}: U_{0} \times\right]-\epsilon, \epsilon[\rightarrow \mathbf{G}$ is a $\mathbf{C}^{k-1}$ diffeomorphism for some $\epsilon>0$. Let now $W_{0}:=\exp _{S}\left(U_{0} \times\right]-\epsilon, \epsilon[)$ and let $\Psi=\left(\Psi_{S}, t\right)$ be the inverse of $\exp _{S}$. We need the following:

Lemma 4.1. There exist an open set $U_{00} \subset U_{0}$ and $\epsilon_{0}$ in $] 0, \epsilon\left[\right.$ such that $p_{0}$ belongs to $U_{00}$ and, if $q$ belongs to $W_{00}=\exp _{S}\left(U_{00} \times\right]-\epsilon_{0}, \epsilon_{0}[)$, then it turns out that $t(q)=\delta^{s}(q)$ is the signed distance from $q$ to $S$, which is realized by $\pm \delta\left(q, \Psi_{S}(q)\right)$.

In rough terms, one can shrink $W_{0}$ (in a controlled way) to a smaller set $W_{00}$ in which $t(y)=\delta^{s}(y)$, whenever $y \in W_{00}$.

Proof of Lemma 4.1. Without loss of generality, we consider points $q$ on the "positive" side of $S$, where $\delta(q)=\delta^{s}(q)$ (and obviously, we can assume that $W_{0}=$ $\exp _{S}\left(U_{0} \times\right] 0, \epsilon[)$. We begin by showing that if $p \neq \Psi_{S}(q)$ lies in $U_{0}$, then $d(q, p)>$ $\delta(q):=\inf \{d(q, r): r \in S\}$. We argue by contradiction. Suppose $d(q, p)=\delta(q) \leq$ $d\left(q, \Psi_{S}(q)\right)$. Now we would like to apply Gauss' Lemma to the CC-sphere $\mathbf{S}(q, d(q, p))$ at the point $p$, but we do not know a priori that the CC-sphere is smooth at $p$.

Let $\gamma$ be a length-minimizing geodesic from $q$ to $p$ and let $q^{\prime}$ be a point on $\gamma$, with $q^{\prime} \neq q$ and $q^{\prime} \neq p$. Consider the CC-sphere $\mathbf{S}\left(q^{\prime}, d\left(q^{\prime}, p\right)\right)$.

By the triangle inequality, $\mathbf{S}\left(q^{\prime}, d\left(q^{\prime}, p\right)\right)$ is contained inside the closure of the ball $B(q, \delta(q))$ and it touches $\mathbf{S}(q, \delta(q))$ at $p$. Now, the arc of the geodesic $\gamma$ from $q^{\prime}$ to $p$, can be extended beyond $p$ to a length-minimizing geodesic $\widetilde{\gamma}$ from $q^{\prime}$ to $p^{\prime}$, where $p^{\prime}$ is not a point of $\gamma$. Our assumption on the group $\mathbf{G}$ implies that the CC-distance from $q^{\prime}$, that is $d\left(q^{\prime}, \cdot\right)$ ( or $\mathbf{G} \ni r \mapsto d\left(q^{\prime}, r\right)$ ), is of class $\mathbf{C}^{2}$ on an open neighborhood of $\widetilde{\gamma}$. Differentiating with respect to the variable $t$ both sides of the identity $t=d\left(q^{\prime}, \gamma(t)\right)$, we get grad $d(\gamma(t)) \neq 0$. We can then apply the Implicit Function Theorem to the function $d\left(q^{\prime}, \cdot\right)$ and obtain that $\mathbf{S}\left(q^{\prime}, d\left(q^{\prime}, p\right)\right)$ is smooth at $p$.

Theorem 1.2 can now be applied to the CC-sphere $\mathbf{S}\left(q^{\prime}, d\left(q^{\prime}, p\right)\right)$. The geodesic $\gamma$ is determined by the values at $p$ of $\nu_{H}^{\mathbf{S}\left(q^{\prime}, d\left(q^{\prime}, p\right)\right)}$ and $\varpi^{\mathbf{S}\left(q^{\prime}, d\left(q^{\prime}, p\right)\right)}$ (where we have made explicit the dependence of the vectors $\nu_{H}$ and $\varpi$ on the hypersurface). In turn, the values of these parameters only depend on the tangent space $T_{p} \mathbf{S}\left(q^{\prime}, d\left(q^{\prime}, p\right)\right)$ to $\mathbf{S}\left(q^{\prime}, d\left(q^{\prime}, p\right)\right)$ at $p$.

On the other hand, note that $T_{p} \mathbf{S}\left(q^{\prime}, d\left(q^{\prime}, p\right)\right)=T_{p} S$, because $\mathbf{S}\left(q^{\prime}, d\left(q^{\prime}, p\right)\right)$ is on one side of $S$, and it is smooth. Since the map $\exp _{S}$ at $p$ only depends on $T_{p} S$, we have that $q=\exp _{S}(p, d(p, q))$.

Recall that $\exp _{S}^{-1}(q)=\left(\Psi_{S}(q), t(q)\right)$. Then we have

$$
\exp _{S}(p, d(p, q))=q=\exp _{S}\left(\Psi_{S}(q), d(q, t(q))\right.
$$

which contradicts the fact that $\exp _{S}$ is diffeomorphic, hence injective, on $\left.U_{0} \times\right]-\epsilon, \epsilon[$. The rest of the argument is purely metric, and it involves no notion of differential geometry.

It might still be possible that there is some $p$ in $S_{0} \backslash U_{0}$ such that

$$
d(q, p)=\delta(q)<d\left(q, \Psi_{S}(q)\right) .
$$

In such a case, the CC-ball $B\left(q, d\left(q, \Psi_{S}(q)\right)\right)$ having center $q$ and radius $d\left(q, \Psi_{S}(q)\right)$ contains $p$ in its interior and so its boundary $\mathbf{S}\left(q, d\left(q, \Psi_{S}(q)\right)\right)$ touches $U_{0}$ at $\Psi_{S}(q)$ only, by the previous case. Let now $q_{1}$ be a point on the geodesic $\gamma$, which joins $q$ and 
$\Psi_{S}(q)$, and consider the metric ball $B\left(q_{1}, d\left(q_{1}, \Psi_{S}\left(q_{1}\right)\right)\right)$. By the triangle inequality,

$$
B\left(q_{1}, d\left(q_{1}, \Psi_{S}\left(q_{1}\right)\right)\right) \subset B\left(q, d\left(q, \Psi_{S}(q)\right)\right)
$$

and the two balls touch at $\Psi_{S}\left(q_{1}\right)=\Psi_{S}(q)$.

Next, we show that, if the number $\epsilon^{\prime}=d\left(q_{1}, \Psi_{S}\left(q_{1}\right)\right)$ is small enough, then we have that $B\left(q_{1}, d\left(q_{1}, \Psi_{S}\left(q_{1}\right)\right)\right)$ is contained in $W_{0}$; hence, it follows that $d\left(q_{1}, \Psi_{S}\left(q_{1}\right)\right)=\epsilon^{\prime}=$ $\delta\left(q_{1}\right)$. To this aim, let

$$
\delta:=\inf \left\{d\left(\Psi_{S}\left(q_{1}\right), r\right): r \in \partial W_{0} \backslash U_{0}\right\}>0
$$

and let $\epsilon^{\prime}<\delta / 2$. Furthermore, let $z$ be a point in $\mathbf{G} \backslash W_{0}, z \neq \Psi_{S}\left(q_{1}\right)$. Consider a geodesic $\sigma$ which minimizes the distance between $z$ and $q_{1}$.

Two cases might occur:

(A) $\sigma$ intersects $U_{0}$ at a point $p_{2}$. Then,

$$
\begin{aligned}
d\left(z, q_{1}\right) & \geq d\left(p_{2}, q_{1}\right) \geq d\left(p_{2}, q\right)-d\left(q_{1}, q\right) \\
& >d\left(\Psi_{S}\left(q_{1}\right), q\right)-d\left(q, q_{1}\right)
\end{aligned}
$$

the strict inequality having been proved before,

$$
=d\left(\Psi_{S}\left(q_{1}\right), q_{1}\right)
$$

because $q, q_{1}, \Psi_{S}(q)=\Psi_{S}(q)$ belong to

the same length-minimizing geodesic $\sigma$

$$
=\epsilon^{\prime}
$$

so that $z \notin B\left(q_{1}, \epsilon^{\prime}\right)$.

(B) $\sigma$ intersects $\partial W_{0} \backslash U_{0}$ at a point $p_{3}$. Then,

$$
d\left(z, q_{1}\right) \geq d\left(p_{3}, q_{1}\right) \geq d\left(p_{3}, \Psi_{S}\left(q_{1}\right)\right)-d\left(\Psi_{S}\left(q_{1}\right), q_{1}\right) \geq \delta-\epsilon^{\prime}>\epsilon^{\prime},
$$

hence $z \notin B\left(q_{1}, \epsilon^{\prime}\right)$.

In particular, as a consequence of the previous argument, we have shown that if $w \in B\left(q_{1}, \epsilon^{\prime}\right)$, then any geodesic joining $w$ and $q_{1}$ does not cross $\partial W_{0}$, hence that $B\left(q_{1}, \epsilon^{\prime}\right) \subset W_{0}$.

To finish the lemma, we have to make the choice of $\epsilon^{\prime}$ quantitative. Let $U_{00}$ be an open set containing $p_{0}$, and compactly contained in $U_{0}$, such that

$$
\eta:=\inf \left\{d(z, w): z \in U_{00}, w \in \partial W_{0} \backslash U_{0}\right\}>0,
$$

and let $\epsilon_{0}:=\eta / 2$. For points $q$ in $\left.U_{00} \times\right]-\epsilon_{0}, \epsilon_{0}$ [ note that our previous argument works and this achieves the proof of Lemma 4.1 .

Roughly speaking, Theorem 1.1 follows from the usual Inverse Function Theorem, applied to the inverse of $\exp _{S}$. More precisely, Corollary 3.3 and Lemma 4.1 together imply that every open set $U_{0}$ compactly contained in $S_{0}$, has an open neighborhood $U:=\exp _{S}\left(U_{0} \times\right]-\epsilon_{0}, \epsilon_{0}[) \subset \mathbf{G}$ satisfying the unique nearest point property (with respect to the CC-distance $d$ ), that is, for every $x \in U$ there exists a unique point $y \in U_{0} \subset S_{0}$ such that $\delta(x)=d(x, y)$. By using the previous notation, one has $\Psi(x)=(y, t)$, where $y=\Psi_{S}(x)$ and $t(x)=\delta^{s}(x)= \pm d(x, y)$.

Thus, let $X \in \mathfrak{X}(\mathbf{G})$ be a smooth vector field on $\mathbf{G}$. Set $y:=\Psi_{S}(x)$, where $\Psi_{S}(x)$ denotes the projection along $U_{0} \subset S_{0}$ of $x \in U:=\Phi\left(U_{0} \times\right]-\epsilon_{0}, \epsilon_{0}[)$. With no loss of generality, we can assume that $x \in B\left(x_{0}, r\right)^{+} \cup\left(U_{0} \cap B\left(x_{0}, r\right)\right)$, where $x_{0} \in U_{0}$ and $r$ 
is sufficiently small. We have

$$
\begin{aligned}
\left\langle\operatorname{grad} \delta^{s}(x), X\right\rangle= & \langle\operatorname{grad} \delta(x), X\rangle \\
= & \left\langle\operatorname{grad}_{x} d\left(x, \Psi_{S}(x)\right), X\right\rangle \\
= & \left\langle\left.\left(\operatorname{grad}_{x} d(x, y)\right)\right|_{y=\Psi_{S}(x)}, X\right\rangle \\
& +\left\langle\left[\mathcal{J}_{x} \Psi_{S}(x)\right] X,\left.\left(\operatorname{grad}_{y} d(x, y)\right)\right|_{y=\Psi_{S}(x)}\right\rangle .
\end{aligned}
$$

Let $\nu^{\mathbf{S}(x, t)}$ be the Riemannian unit outward normal to the CC-sphere $\mathbf{S}(x, t)$ at any smooth point $y \in \mathbf{S}(x, t)$. Below we shall set

$$
\mathcal{N}_{x, t}(y):=\frac{\nu^{\mathbf{S}(x, t)}(y)}{\left|\mathcal{P}_{H}\left(\nu^{\mathbf{S}(x, t)}(y)\right)\right|}
$$

By our Gauss' Lemma (see Theorem 1.2), we immediately get that

$$
\operatorname{grad}_{x} d(x, y)=\mathcal{N}_{y, t}(x), \quad \operatorname{grad}_{y} d(x, y)=\mathcal{N}_{x, t}(y) .
$$

Applying Gauss' Lemma and recalling the explicit form of normal geodesics in 2-step Carnot groups yields

$$
\mathcal{N}_{y, t}(x)=\left(e^{-C_{H}(\varpi(y)) t} \nu_{H}(y), \varpi(y)\right) .
$$

Notice that $\mathcal{N}_{y, t}$ is of class $\mathbf{C}^{k-1}$ as well as $\mathcal{N}=\left(\nu_{H}, \varpi\right)$. Furthermore, $\mathcal{N}_{x, t}(y)=$ $\pm \mathcal{N}(y)$, where the sign only depends on the given orientation of $S$. By the previous discussion and (19) we get that

$$
\langle\operatorname{grad} \delta(x), X\rangle=\left\langle\mathcal{N}_{\Psi_{S}(x), t}(x), X\right\rangle+\left\langle\left[\mathcal{J}_{x} \Psi_{S}(x)\right] X, \mathcal{N}_{x, t}\left(\Psi_{S}(x)\right)\right\rangle .
$$

Since $\left[\mathcal{J}_{x} \Psi_{S}(x)\right] X \in T_{\Psi_{S}(x)} S$, by using the fact that $\mathcal{N}_{x, t}\left(\Psi_{S}(x)\right)$ is normal to $S$ at $\Psi_{S}(x)$ one gets

$$
\left\langle\left[\mathcal{J}_{x} \Psi_{S}(x)\right] X, \mathcal{N}_{x, t}\left(\Psi_{S}(x)\right)\right\rangle=0 .
$$

Thus, we have $\langle\operatorname{grad} \delta(x), X\rangle=\left\langle\mathcal{N}_{\Psi_{S}(x), t}(x), X\right\rangle$ and, by the arbitrariness of $X \in$ $\mathfrak{X}(\mathbf{G})$, it follows that grad $\delta$ is of class $\mathbf{C}^{k-1}$ on $U \backslash U_{0}$. Hence $\delta$ is of class $\mathbf{C}^{k}$ on $B\left(x_{0}, r\right)^{+} \cup\left(U_{0} \cap B\left(x_{0}, r\right)\right)$ and hence on $U$, as desired.

\section{References}

[1] Agrachev, A.: Any sub-Riemannian metric has points of smoothness. - Dokl. Akad. Nauk 424:3, 2009, 295-298; Engl. transl. in Dokl. Math. 79:1, 2009, 45-47.

[2] Agrachev, A., U. Boscain, and D. Barillari: Introduction to Riemannian and sub-Riemannian geometry. - Lecture notes, http://people.sissa.it/agrachev/agrachev_files/ notes.html.

[3] Arcozzi, N., and F. Ferrari: Metric normal and distance function in the Heisenberg group. - Math. Zeit. 256, 2007, 661-684.

[4] Arcozzi, N., and F. Ferrari: The Hessian of the distance from a surface in the Heisenberg group. - Ann. Acad. Sci. Fenn. Math. 33, 2008, 35-63.

[5] Balogh, Z. M., C. Pintea, and H. Rohner: Size of tangencies to non-involutive distributions. - Indiana Univ. Math. J. 60:6, 2011, 2061-2092.

[6] Bellä̈Che, A.: The tangent space in subriemannian geometry. - In: Sub-Riemannian geometry, Progr. Math. 144, edited by A. Bellaïche and J. Risler, Birkhauser Verlag, Basel, 1996.

[7] Chavel, I: Riemannian geometry: a modern introduction. - Cambridge Univ. Press, 1994.

[8] Federer, H.: Curvature measures. - Trans. Amer. Math. Soc. 93:3, 1959, 418-491. 
[9] Foote, R. L.: Regularity of the distance function. - Proc. Amer. Math. Soc. 92:1, 1984.

[10] Garofalo, N., and D. M. Nhieu: Isoperimetric and Sobolev inequalities for CarnotCarathéodory spaces and the existence of minimal surfaces. - Comm. Pure Appl. Math. 49, 1996, 1081-1144.

[11] Gromov, M.: Carnot-Carathéodory spaces seen from within. - In: Subriemannian geometry, Progr. Math. 144, edited by A. Bellaïche and J. Risler, Birkhauser Verlag, Basel, 1996.

[12] Gilbarg, D., and N. Trudinger: Elliptic partial differential equations of second order. Springer Verlag, Berlin, 1977.

[13] Golé, C., and R. KARIDI: A note on Carnot geodesics in nilpotent Lie groups. - J. Dyn. Control Syst. 1, 1995, 535-549.

[14] Krantz, S. G., and H. R. Parks: Distance to $\mathbf{C}^{k}$ hypersurfaces. - J. Differentail Equations 40, 1981, 116-120.

[15] Le Donne, E., and S. Nicolussi Golo: Regularity properties of spheres in homogeneous groups. - Preprint, 2015.

[16] LeE, J. M.: Introduction to smooth manifolds. - Springer Verlag, 2003.

[17] Magnani, V.: Elements of geometric measure theory on sub-Riemannian groups. - PhD Thesis, Scuola Normale Superiore di Pisa, 2002.

[18] Montefalcone, F.: Hypersurfaces and variational formulas in sub-Riemannian Carnot groups. - J. Math. Pures Appl. 87, 2007, 453-494.

[19] Monti, R.: Some properties of Carnot-Carathéodory balls in the Heisenberg group. - Atti Accad. Naz. Lincei Cl. Sci. Fis. Mat. Natur. Rend. Lincei (9) Mat. Appl. 11:3, 2001, 155-167.

[20] Monti, R., and F. Serra Cassano: Surface measures in Carnot-Carathéodory spaces. Calc. Var. Partial Differential Equations 13, 2001, 339-376.

[21] Montgomery, R.: A tour of subriemannian geometries, their geodesics and applications. Math. Surveys Monogr. 91, Amer. Math. Soc., 2002.

[22] Pansu, P.: Métriques de Carnot Carathéodory et quasi-isométries des espaces symmétriques de rang un. - Ann. of Math. (2) 129, 1989, 1-60.

[23] Paulat, M.: Sub-Riemannian geometry and heat kernel estimates. - Dissertation, C. A. University Kiel, 2008.

[24] Rifford, L.: À propos des sphères sous-riemanniennes. - Bull. Belg. Math. Soc. Simon Stevin $13: 3,2006,521-526$.

[25] Rifford, L., and E. Trélat: Morse-Sard type results in sub-Riemannian geometry. - Math. Ann. 332:1, 2005, 145-159.

[26] Strichartz, R. S.: Sub-Riemannian geometry. - J. Diff. Geom. 24, 1986, 221-263; Corrections in J. Diff. Geom. 30, 1989, 595-596.

[27] Tyson, J.: Global conformal Assouad dimension in the Heisenberg group. - Conform. Geom. Dyn. $12,2008,32-57$.

[28] Varadarajan, V. S.: Lie groups, Lie algebras and their representations. Reprint of the 1974 edition. - Grad. Texts in Math. 102, Springer, New York, NY, 1984.

[29] Vershik, A. M., and V. Ya. Gershkovich: Nonholonomic dynamical systems, geometry of distributions and variationals problems. - In: Dynamical systems VII, edited by V. I. Arnold and S. P. Novikov, Springer-Verlag, 1996.

Received 2 February $2015 \bullet$ Revised received 8 July $2016 \bullet$ Accepted 2 September 2016 